\title{
Repdigits Base $b$ as Difference of Two Fibonacci Numbers
}

\author{
Zafer Şiar ${ }^{1}$, Fatih Erduvan ${ }^{2}$ and Refik Keskin ${ }^{2, *}$ \\ ${ }^{1}$ Bingöl University, Department of Mathematics, Bingöl 12000, Turkey. \\ 2 Sakarya University, Department of Mathematics, Sakarya TR-54187, Turkey.
}

Received February 21, 2020; Accepted May 10, 2021;

Published online January 18, 2022.

$$
\begin{aligned}
& F_{9}-F_{4}=34-3=31=(11111)_{2}, \quad F_{14}-F_{7}=377-13=364=(111111)_{3}, \\
& F_{14}-F_{7}=377-13=364=(222)_{4}, \quad F_{9}-F_{4}=34-3=31=(111)_{5} \text {, } \\
& F_{11}-F_{4}=89-3=86=(222)_{6}, \quad F_{13}-F_{5}=233-5=228=(444)_{7}, \\
& F_{10}-F_{2}=55-1=54=(66)_{8}, \quad F_{14}-F_{7}=377-13=364=(444)_{9} \text {, }
\end{aligned}
$$

and

$$
F_{15}-F_{10}=610-55=555=(555)_{10}
$$

As a result, it is shown that the largest Fibonacci number which can be written as a sum of a repdigit and a Fibonacci number is $F_{15}=610=555+55=555+F_{10}$.

AMS subject classifications: 11B39, 11J86, 11D61

Key words: Fibonacci numbers, repdigit, Diophantine equations, linear forms in logarithms.

\section{Introduction}

Let $\left(F_{n}\right)$ and $\left(L_{n}\right)$ be the sequences of Fibonacci and Lucas numbers given by $F_{0}=0, F_{1}=1$, $L_{0}=2, L_{1}=1, F_{n}=F_{n-1}+F_{n-2}$ and $L_{n}=L_{n-1}+L_{n-2}$ for $n \geq 2$, respectively. Binet formulas for these numbers are $F_{n}=\frac{\alpha^{n}-\beta^{n}}{\sqrt{5}}$ and $L_{n}=\alpha^{n}+\beta^{n}$, where $\alpha=\frac{1+\sqrt{5}}{2}$ and $\beta=\frac{1-\sqrt{5}}{2}$, which are the roots of the characteristic equation $x^{2}-x-1=0$. It can be seen that $1<\alpha<2,-1<\beta<0$

*Corresponding author. Email addresses: zsiar@bingol .edu.tr (Şiar Z), erduvanmat@hotmail.com.tr (Erduvan F), keskin@sakarya. edu.tr (Keskin R) 
and $\alpha \beta=-1$. For more about Fibonacci and Lucas numbers with their applications, one can see [9]. The relation between Fibonacci number $F_{n}$ and $\alpha$ are given by

$$
\alpha^{n-2} \leq F_{n} \leq \alpha^{n-1}
$$

for $n \geq 1$. The inequality (1.1) can be proved by induction. A repdigit in base $b$ is a positive integer $N$ whose digits are all equal. If $b=10$, we say that $N$ is a repdigit. Recently, the problem of finding the repdigits in the second-order linear recurrence sequences has been of interest to mathematicians. In [10], Luca has found all repdigits in the Fibonacci and Lucas sequences. The largest repdigits in Fibonacci and Lucas sequences are $F_{10}=55$ and $L_{5}=11$. Luca [11] also found all repdigits which are sums of three Fibonacci numbers. In [6], Erduvan and Keskin showed that if $F_{m} F_{n}$ is a repdigit in base 10, then $F_{m} F_{n} \in\{1,2,3,4,5,6,8,9,55\}$. Later Keskin, Erduvan and Siar [7] have found that if $F_{m} F_{n}$ is a repdigit in base $b$, and has at least two digits, then $F_{m} F_{n} \in\{3,4,5,6,8,9,10,13,15,16,21,24,26,40,42,63,170,273\}$, where $b=2,3,4,5,6,7,8,9$ and $2 \leq m \leq n$. Motivated by the above studies, here, we consider the Diophantine equation

$$
F_{n}-F_{m}=N=\frac{d \cdot\left(b^{k}-1\right)}{b-1}
$$

where $2 \leq b \leq 10,2 \leq m<n, 1 \leq d \leq b-1$ and $k \geq 2$. Since the values $F_{1}$ and $F_{2}$ are the same, we start this equation from $m=2$ instead of $m=1$. In Section 2, we introduce necessary lemmas and theorems. Then in Section 3, we prove our main results (Theorem 3.1) on the solutions to Eq. (1.2).

\section{Auxiliary results}

In order to solve Diophantine equations of the form (1.2), we use Baker's theory for lower bounds for a nonzero linear form in logarithms of algebraic numbers. Since such bounds are very important in effectively solving of Diophantine equations, we start with recalling some basic notions from algebraic number theory.

Let $\eta$ be an algebraic number of degree $d$ with the minimal polynomial

$$
a_{0} x^{d}+a_{1} x^{d-1}+\ldots+a_{d}=a_{0} \prod_{i=1}^{d}\left(x-\eta^{(i)}\right) \in \mathbb{Z}[x],
$$

where the $a_{i}$ 's are relatively prime integers with $a_{0}>0$ and the $\eta^{(i)}$ 's are conjugates of $\eta$. Then

$$
h(\eta)=\frac{1}{d}\left(\log a_{0}+\sum_{i=1}^{d} \log \left(\max \left\{\left|\eta^{(i)}\right|, 1\right\}\right)\right)
$$

is called the logarithmic height of $\eta$. If $\eta=a / b$ is a rational number with $\operatorname{gcd}(a, b)=1$ and $b \geq 1$, then $h(\eta)=\log (\max \{|a|, b\})$. 
The following properties of logarithmic height are found in several works stated in references:

$$
\begin{aligned}
& h(\eta \pm \gamma) \leq h(\eta)+h(\gamma)+\log 2, \\
& h\left(\eta \gamma^{ \pm 1}\right) \leq h(\eta)+h(\gamma), \\
& h\left(\eta^{k}\right)=|k| h(\eta) .
\end{aligned}
$$

The following theorem can be deduced from Corollary 2.3 of Matveev [12], which provides a large upper bound for the subscript $n$ in the equation (1.2) (also see Theorem 9.4 in [3]).

Theorem 2.1. Assume that $\gamma_{1}, \gamma_{2}, \ldots, \gamma_{t}$ are positive real algebraic numbers in a real algebraic number field $\mathbb{K}$ of degree $D, b_{1}, b_{2}, \ldots, b_{t}$ are rational integers, and

$$
\Lambda:=\gamma_{1}^{b_{1}} \cdots \gamma_{t}^{b_{t}}-1
$$

is not zero. Then

$$
|\Lambda|>\exp \left(-1.4 \cdot 30^{t+3} \cdot t^{4.5} \cdot D^{2}(1+\log D)(1+\log B) A_{1} A_{2} \cdots A_{t}\right),
$$

where

$$
B \geq \max \left\{\left|b_{1}\right|, \ldots,\left|b_{t}\right|\right\},
$$

and

$$
A_{i} \geq \max \left\{D h\left(\gamma_{i}\right),\left|\log \gamma_{i}\right|, 0.16\right\}, \quad \text { for } i=1, \ldots, t .
$$

The following lemma is given in [2]. This lemma is an immediate variation of the result due to Dujella and Pethő from [5], which is a version of a lemma of Baker and Davenport [1]. This lemma will be used to reduce the upper bound for the subscript $n$ in the equation (1.2). For a real number $x,\|x\|$ denotes the distance from $x$ to the nearest integer. That is, ||$x||=\min \{|x-n|: n \in \mathbb{Z}\}$.

Lemma 2.1. Let $M$ be a positive integer, let $p / q$ be a convergent of the continued fraction of the irrational number $\gamma$ such that $q>6 M$, and let $A, B, \mu$ be some real numbers with $A>0$ and $B>1$. Let $\epsilon:=|| \mu q\|-M\| \gamma q \|$. If $\epsilon>0$, then there exists no solution to the inequality

$$
0<|u \gamma-v+\mu|<A B^{-w}
$$

in positive integers $u, v$, and $w$ with $u \leq M$ and $w \geq \frac{\log (A q / \epsilon)}{\log B}$.

Now we give a lemma which can be proved by using Binet's formulae.

Lemma 2.2. Assume that $n \equiv m(\bmod 2)$. Then

$$
F_{n}-F_{m}= \begin{cases}F_{(n-m) / 2} L_{(n+m) / 2} & \text { if } n \equiv m(\bmod 4), \\ F_{(n+m) / 2} L_{(n-m) / 2} & \text { if } n \equiv m+2(\bmod 4) .\end{cases}
$$


We need to following result in [4].

Lemma 2.3. Let $a, x \in \mathbb{R}$. If $0<a<1$ and $|x|<a$, then

$$
|\log (1+x)|<\frac{-\log (1-a)}{a} \cdot|x|
$$

and

$$
|x|<\frac{a}{1-e^{-a}} \cdot\left|e^{x}-1\right| .
$$

The following theorem is a result of the combination of the theorems in [6] and [7].

Theorem 2.2. Let $2 \leq m \leq n$ and $b$ be a positive integer such that $2 \leq b \leq 10$. If $N=F_{n} F_{m}$ is $a$ repdigit in base $b$ and has at least two digits, then

$$
(n, m, b, d, k, N) \in\left\{\begin{array}{c}
(3,3,3,1,2,4),(4,2,2,1,2,3),(4,3,5,1,2,6), \\
(4,4,8,1,2,9),(5,2,4,1,2,5),(5,3,4,2,2,10), \\
(5,3,9,1,2,10),(5,4,4,3,2,15),(5,4,2,1,4,15), \\
(6,2,3,2,2,8),(6,2,7,1,2,8),(6,3,7,2,2,16), \\
(8,4,2,1,6,63),(6,4,7,3,2,24),(6,5,7,5,2,40), \\
(6,5,9,4,2,40),(6,5,3,1,4,40),(7,3,3,2,3,26), \\
(7,2,3,1,3,13),(8,2,6,3,2,21),(8,2,4,1,3,21), \\
(8,3,4,2,3,42),(8,4,8,7,2,63),(10,2,10,5,2,55), \\
(8,4,4,3,3,63),(8,7,9,3,3,273),(9,5,4,2,4,170)
\end{array}\right\} .
$$

The following theorem is a result of the combination of Theorem 6 given in [6] and Theorem 4 given in [8].

Theorem 2.3. Let $0 \leq m \leq n$ and $b$ be a positive integer such that $2 \leq b \leq 10$. If $N=L_{n} L_{m}$ is $a$ repdigit in base $b$ and has at least two digits, then

$$
(n, m, b, d, k, N) \in\left\{\begin{array}{c}
(0,0,3,1,2,4),(3,0,3,2,2,8),(3,0,7,1,2,8), \\
(4,0,6,2,2,14),(2,0,5,1,2,6),(3,1,3,1,2,4), \\
(4,1,6,1,2,7),(4,1,2,1,3,7),(2,1,2,1,2,3), \\
(2,2,8,1,2,9),(6,0,8,4,2,36),(6,1,5,3,2,18), \\
(6,1,8,2,2,18),(3,2,5,2,2,12),(4,2,4,1,3,21), \\
(4,2,6,3,2,21),(6,2,8,6,2,54),(9,2,7,4,3,228), \\
(3,3,7,2,2,16),(4,3,6,4,2,28),(5,5,3,1,5,121), \\
(5,0,10,2,2,22),(5,1,10,1,2,11),(5,2,10,3,2,33), \\
(5,3,10,4,2,44),(5,4,10,7,2,77)
\end{array}\right\} .
$$




\section{Main theorem}

Theorem 3.1. Let $2 \leq b \leq 10,2 \leq m<n, k \geq 2$ and $1 \leq d \leq b-1$. The only solutions of the Diophantine equation (1.2) in nonnegative integers are given by

$$
(n, m, b, d, k, N) \in\left\{\begin{array}{c}
(5,2,3,1,2,4),(5,3,2,1,2,3),(6,2,6,1,2,7), \\
(6,2,2,1,3,7),(6,3,5,1,2,6),(6,4,4,1,2,5), \\
(6,5,2,1,2,3),(7,2,5,2,2,12),(7,3,10,1,2,11) \\
(7,4,4,2,2,10),(7,4,9,1,2,10),(7,5,3,2,2,8), \\
(7,5,7,1,2,8),(7,6,4,1,2,5),(8,2,9,2,2,20) \\
(8,4,5,3,2,18),(8,4,8,2,2,18),(8,5,7,2,2,16), \\
(8,6,3,1,3,13),(8,7,3,2,2,8),(8,7,7,1,2,8), \\
(9,2,10,3,2,33),(9,3,7,4,2,32),(9,4,5,1,3,31), \\
(9,4,2,1,5,31),(9,6,3,2,3,26),(9,7,6,3,2,21), \\
(9,7,4,1,3,21),(9,8,3,1,3,13),(10,2,8,6,2,54), \\
(10,5,9,5,2,50),(10,7,4,2,3,42),(10,9,6,3,2,21), \\
(10,9,4,1,3,21),(11,2,10,8,2,88),(11,4,6,2,3,86), \\
(11,9,10,5,2,55),(12,11,10,5,2,55),(13,5,7,4,3,228) \\
(14,7,9,4,3,364),(14,7,3,1,6,364),(15,10,10,5,3,555)
\end{array}\right\} .
$$

Proof. Assume that the equation (1.2) holds. Let $n-m=1$. Then, we get

$$
F_{m-1}=\frac{d \cdot\left(b^{k}-1\right)}{b-1}
$$

By Theorem 2.2, we have

$$
(n, m, b, d, k, N) \in\left\{\begin{array}{c}
(6,5,2,1,2,3),(7,6,4,1,2,5),(8,7,3,2,2,8) \\
(8,7,7,1,2,8),(9,8,3,1,3,13),(10,9,6,3,2,21) \\
(10,9,4,1,3,21),(12,11,10,5,2,55)
\end{array}\right\}
$$

With the help of Mathematica program, we obtain the solutions displayed in Theorem 3.1 for $2 \leq m<n \leq 149$.

From now on, assume that $n \geq 150, m \geq 2$, and $n-m \geq 2$. Using the identity (1.1), we get the inequality

$$
2^{k-1} \leq b^{k-1}<\frac{d \cdot\left(b^{k}-1\right)}{b-1}=F_{n}-F_{m}<F_{n} \leq \alpha^{n-1}<2^{n-1},
$$

which shows that $k<n$. Rearranging the equation (1.2) as

$$
\frac{\alpha^{n}}{\sqrt{5}}-\frac{d \cdot b^{k}}{b-1}=F_{m}+\frac{\beta^{n}}{\sqrt{5}}-\frac{d}{b-1}
$$


and taking absolute values, we obtain

$$
\left|\frac{\alpha^{n}}{\sqrt{5}}-\frac{d \cdot b^{k}}{b-1}\right|=\left|F_{m}+\frac{\beta^{n}}{\sqrt{5}}-\frac{d}{b-1}\right| \leq F_{m}+\frac{|\beta|^{n}}{\sqrt{5}}+\frac{d}{b-1}<\alpha^{m-1}+1.1
$$

by the inequality (1.1). Dividing both sides of the above inequality by $\frac{\alpha^{n}}{\sqrt{5}}$, we get

$$
\begin{aligned}
\left|1-\frac{\alpha^{-n} \sqrt{5} \cdot d \cdot b^{k}}{b-1}\right| & <\sqrt{5} \alpha^{m-n-1}+1.1 \sqrt{5} \alpha^{-n} \\
& =\sqrt{5} \alpha^{m-n}\left(\frac{1}{\alpha}+1.1 \alpha^{-m}\right)<\frac{2.33}{\alpha^{n-m}}
\end{aligned}
$$

where we have used the fact that $m \geq 2$. Next we shall apply Theorem 2.1 with

$$
\gamma_{1}:=b, \gamma_{2}:=\alpha, \gamma_{3}:=\frac{d \cdot \sqrt{5}}{(b-1)} \text { and } b_{1}:=k, b_{2}:=-n, b_{3}:=1 \text {. }
$$

Note that the numbers $\gamma_{1}, \gamma_{2}$, and $\gamma_{3}$ are positive real numbers and elements of the field $\mathbb{K}=\mathbb{Q}(\sqrt{5})$, so $D=2$. We show that $\Lambda_{1}:=\frac{\alpha^{-n} \sqrt{5} \cdot d \cdot b^{k}}{b-1}-1$ is nonzero. For, if $\Lambda_{1}=0$, then we get $\alpha^{n}=\frac{\sqrt{5} \cdot d \cdot b^{k}}{b-1}$. Conjugating in $\mathbb{Q}(\sqrt{5})$ gives us $\beta^{n}=\frac{-\sqrt{5} \cdot d \cdot b^{k}}{b-1}$ and so $L_{n}=\alpha^{n}+\beta^{n}=0$, which is impossible. Moreover, since

$$
h\left(\gamma_{1}\right)=h(b)=\log b \leq \log 10<2.31, \quad h\left(\gamma_{2}\right)=h(\alpha)=\frac{\log \alpha}{2}=\frac{0.4812 \ldots}{2},
$$

and

$$
h\left(\gamma_{3}\right)=h\left(\frac{d \cdot \sqrt{5}}{b-1}\right) \leq h(d)+h(\sqrt{5})+h(b-1) \leq 2 \log 9+\frac{\log 5}{2}<5.2
$$

by the identity (2.1), we can take $A_{1}:=4.62, A_{2}:=0.5$, and $A_{3}:=10.4$. Since $k<n$, it follows that

$$
B:=n \geq \max \left\{\left|b_{1}\right|,\left|b_{2}\right|,\left|b_{3}\right|\right\}=\max \{|k|,|-n|, 1\} .
$$

Thus, taking into account the inequality (3.1) and using Theorem 2.1, we obtain

$$
\frac{2.33}{\alpha^{n-m}}>\left|\Lambda_{1}\right|>\exp \left(-1.4 \cdot 30^{6} \cdot 3^{4.5} \cdot 2^{2}(1+\log 2)(1+\log n)(4.62)(0.5)(10.4)\right) .
$$

From the last inequality, a quick computation with Mathematica gives us the inequality

$$
(n-m) \log \alpha-\log (2.33)<(1+\log n) \cdot(2.33) \cdot 10^{13} .
$$

In what follows, we shall apply Theorem 2.1 again. Rearranging Eq. (1.2) as

$$
\frac{\alpha^{n}}{\sqrt{5}}-\frac{\alpha^{m}}{\sqrt{5}}-\frac{d \cdot b^{k}}{b-1}=\frac{\beta^{n}}{\sqrt{5}}-\frac{\beta^{m}}{\sqrt{5}}-\frac{d}{b-1}
$$


and taking absolute values, we obtain

$$
\left|\frac{\alpha^{n}\left(1-\alpha^{m-n}\right)}{\sqrt{5}}-\frac{d \cdot b^{k}}{b-1}\right|=\left|\frac{\beta^{n}}{\sqrt{5}}-\frac{\beta^{m}}{\sqrt{5}}-\frac{d}{b-1}\right| \leq \frac{|\beta|^{n}+|\beta|^{m}}{\sqrt{5}}+\frac{d}{b-1}<1.18,
$$

where we used the fact that $|\beta|^{n}+|\beta|^{m}<0.4$ for $n \geq 150$ and $m \geq 2$. Dividing both sides of the above inequality by

$$
\frac{\alpha^{n}\left(1-\alpha^{m-n}\right)}{\sqrt{5}}
$$

we get

$$
\left|1-\frac{\alpha^{-n} \cdot b^{k} \cdot d \cdot \sqrt{5} \cdot\left(1-\alpha^{m-n}\right)^{-1}}{b-1}\right|<(1.18) \cdot \sqrt{5} \alpha^{-n}\left(1-\alpha^{m-n}\right)^{-1} .
$$

Since

$$
\alpha^{m-n}=\frac{1}{\alpha^{n-m}} \leq \frac{1}{\alpha}<0.62
$$

it is seen that

$$
1-\alpha^{m-n}>0.38
$$

and therefore

$$
\frac{1}{1-\alpha^{m-n}}<\frac{100}{38}
$$

Then from (3.3), it follows that

$$
\left|1-\frac{\alpha^{-n} \cdot b^{k} \cdot d \cdot \sqrt{5} \cdot\left(1-\alpha^{m-n}\right)^{-1}}{b-1}\right|<\frac{5.89}{\alpha^{n}} .
$$

Thus, taking

$$
\gamma_{1}:=b, \quad \gamma_{2}:=\alpha, \quad \gamma_{3}:=\frac{d \cdot \sqrt{5} \cdot\left(1-\alpha^{m-n}\right)^{-1}}{b-1}
$$

and $b_{1}:=k, b_{2}:=-n, b_{3}:=1$, we can apply Theorem 2.1. The numbers $\gamma_{1}, \gamma_{2}$, and $\gamma_{3}$ are positive real numbers and elements of the field $\mathbb{K}=\mathbb{Q}(\sqrt{5})$, so $D=2$. We now show that

$$
\Lambda_{2}:=\frac{\alpha^{-n} \cdot b^{k} \cdot d \cdot \sqrt{5} \cdot\left(1-\alpha^{m-n}\right)^{-1}}{b-1}-1
$$

is nonzero. Suppose $\Lambda_{2}=0$. Then

$$
\alpha^{n}=\frac{b^{k} \cdot d \cdot \sqrt{5} \cdot\left(1-\alpha^{m-n}\right)^{-1}}{b-1} .
$$

Conjugating in $\mathbb{Q}(\sqrt{5})$ gives us

$$
\beta^{n}=\frac{-b^{k} \cdot d \cdot \sqrt{5} \cdot\left(1-\beta^{m-n}\right)^{-1}}{b-1} .
$$


By a simple computation, it is seen that $L_{n}=L_{m}$, which is impossible since $n>m$. As

$$
h\left(\gamma_{1}\right)=h(b) \leq \log 10<2.31 \text { and } h\left(\gamma_{2}\right)=h(\alpha)=\frac{\log \alpha}{2}=\frac{0.4812 \ldots}{2}
$$

by (2.1), we can take $A_{1}:=4.62$ and $A_{2}:=0.5$. On the other hand, using (2.2), (2.3), and (2.4), we get

$$
\begin{aligned}
h\left(\gamma_{3}\right) & =h\left(\frac{d \cdot \sqrt{5} \cdot\left(1-\alpha^{m-n}\right)^{-1}}{b-1}\right) \\
& \leq h(d)+h(\sqrt{5})+h(b-1)+(n-m) h(\alpha)+\log 2 \\
& \leq 2 \log 9+\log \sqrt{5}+(n-m) \frac{\log \alpha}{2}+\log 2<5.9+(n-m) \frac{\log \alpha}{2} .
\end{aligned}
$$

A simple computation shows that $\left|\log \gamma_{3}\right|<\log 5+(n-m) \log \alpha$. So we can take $A_{3}:=12+$ $(n-m) \log \alpha$. Also, since $k<n$, it follows that $B:=n \geq \max \left\{\left|b_{1}\right|,\left|b_{2}\right|,\left|b_{3}\right|\right\}=\max \{|k|,|-n|, 1\}$. Thus, taking into account the inequality (3.4) and using Theorem 2.1, we obtain

$$
\frac{5.89}{\alpha^{n}}>\left|\Lambda_{2}\right|>\exp ((-C)(1+\log n)(12+(n-m) \log \alpha)),
$$

or

$$
n \log \alpha-\log (5.89)<C(1+\log n)(12+(n-m) \log \alpha),
$$

where $C=1.4 \cdot 30^{6} \cdot 3^{4.5} \cdot 2^{2} \cdot(1+\log 2)(0.5)(4.62)$. Inserting the bound for $(n-m) \log \alpha$ from (3.2) to (3.5), we get

$$
n \log \alpha-\log (5.89)<C(1+\log n)\left(12+(2.33) \cdot 10^{13} \cdot(1+\log n)+\log (2.33)\right) .
$$

With the help of Mathematica, it is seen that $n<5.23 \cdot 10^{29}$.

Next we will further reduce the range of $n$ with the help of Lemma 2.1. Let

$$
z_{1}:=\log \left(\Lambda_{1}+1\right)=k \log b-n \log \alpha+\log \frac{\sqrt{5} \cdot d}{b-1}
$$

From (3.1), we have

$$
\left|\Lambda_{1}\right|=\left|e^{z_{1}}-1\right|<\frac{2.33}{\alpha^{n-m}}<0.9
$$

for $n-m \geq 2$. Choosing $a:=0.9$, we get the inequality

$$
\left|z_{1}\right|=\left|\log \left(\Lambda_{1}+1\right)\right|<\frac{\log 10}{0.9} \cdot \frac{2.33}{\alpha^{n-m}}<\frac{5.97}{\alpha^{n-m}}
$$

by Lemma 2.3. Dividing this inequality by $\log \alpha$, we get

$$
0<\left|k\left(\frac{\log b}{\log \alpha}\right)-n+\left(\frac{\log \frac{\sqrt{5} \cdot d}{b-1}}{\log \alpha}\right)\right|<(12.41) \cdot \alpha^{-(n-m)} .
$$


Now we are in a position to apply Lemma 2.1 with

$$
\gamma:=\frac{\log b}{\log \alpha} \notin \mathbb{Q}, \mu:=\frac{\log \frac{\sqrt{5} \cdot d}{b-1}}{\log \alpha}, A:=12.41, B:=\alpha, \text { and } w:=n-m .
$$

Let $M:=5.23 \cdot 10^{29}$. By using Mathematica, it is seen that denominator $q_{69}$ of the $69^{\text {th }}$ convergent of $\gamma$ exceeds $6 M$. Furthermore,

$$
\epsilon:=\| \mu q_{69}||-M|| \gamma q_{69}||>0.022 \text {. }
$$

Thus, by Lemma 2.1 the inequality (3.7) cannot hold for positive integers $k$ and $n$ with

$$
\frac{\log \left(A q_{69} / \epsilon\right)}{\log B} \leq 186.711 \leq n-m
$$

So it has to be that $n-m \leq 186$. Substituting this upper bound for $n-m$ into (3.5), we obtain $n<1.82 \cdot 10^{16}$.

Next we shall apply Lemma 2.1 to further reduce a little bit the upper bound on $n$. Let

$$
z_{2}:=\log \left(\Lambda_{2}+1\right)=k \log b-n \log \alpha+\log \left(\frac{d \cdot \sqrt{5} \cdot\left(1-\alpha^{m-n}\right)^{-1}}{b-1}\right) .
$$

It is clear that

$$
\left|\Lambda_{2}\right|=\left|e^{z_{2}}-1\right|<(5.89) \cdot \alpha^{-n}<\frac{1}{4}
$$

by (3.4), where we have used the assumption that $n \geq 150$. Thus, taking $a:=0.25$ in Lemma 2.3 and making necessary calculations, we get

$$
\left|z_{2}\right|=\left|\log \left(1+\Lambda_{2}\right)\right|<\frac{\log (4 / 3)}{0.25} \cdot(5.89) \cdot \alpha^{-n}<(6.78) \cdot \alpha^{-n} .
$$

Dividing both sides of the above inequality by $\log \alpha$, we get

$$
0<\left|k\left(\frac{\log b}{\log \alpha}\right)-n+\frac{\log \left(\frac{d \cdot \sqrt{5} \cdot\left(1-\alpha^{m-n}\right)^{-1}}{b-1}\right)}{\log \alpha}\right|<(14.09) \cdot \alpha^{-n} .
$$

Let $\gamma=\frac{\log b}{\log \alpha}$ and $M=1.85 \cdot 10^{16}$. By using Mathematica, it is seen that denominator $q_{43}$ of the $43^{\text {th }}$ convergent of $\gamma$ exceeds $6 M$. Also, taking

$$
\mu=\frac{\log \left(\frac{d \cdot \sqrt{5} \cdot\left(1-\alpha^{m-n}\right)^{-1}}{b-1}\right)}{\log \alpha}
$$

with $n-m \in[1,186]$ and $n-m \notin\{4,8,12\}$, a quick computation with Mathematica gives us the inequality

$$
\epsilon=\| \mu q_{43}||-M|| \gamma q_{43}||>0.00007
$$


Note that when $n-m=4,8,12, \epsilon<0$ for some values of $b$ and $d$. So we cannot apply Lemma 2.1. Let $A=14.09, B=\alpha$, and $w=n$. Using Lemma 2.1, we see that the inequality (3.8) has no solution when

$$
\frac{\log \left(A q_{43} / \epsilon\right)}{\log B} \leq 135.641 \leq n
$$

with $n-m \notin\{4,8,12\}$. Hence we must have $n \leq 135$ with $n-m \notin\{4,8,12\}$. Yet this is impossible as we assumed $n \geq 150$.

Now, we examine the cases $n-m=4,8,12$. If $n-m=4$, we have $F_{n}-F_{m}=L_{m+2}=\frac{d \cdot\left(b^{k}-1\right)}{b-1}$ from (1.2) and Lemma 2.2. This implies that

$$
(n, m, b, d, k, N) \in\left\{\begin{array}{c}
(6,2,6,1,2,7),(6,2,2,1,3,7), \\
(8,4,5,3,2,18),(8,4,8,2,2,18), \\
(7,3,10,1,2,11)
\end{array}\right\}
$$

by Theorem 2.3. If $n-m=8$, then by Lemma 2.2 , we have $F_{n}-F_{m}=L_{2} L_{m+4}$ and so

$$
L_{2} L_{m+4}=\frac{d \cdot\left(b^{k}-1\right)}{b-1}
$$

by (1.2). This shows that

$$
(n, m, b, d, k, N) \in\{(10,2,8,6,2,54),(13,5,7,4,3,228)\}
$$

by Theorem 2.3. If $n-m=12$, then $\epsilon<0$ for $(b, d)=(8,7),(2,1)$. In this case, by Lemma 2.2, we get $8 L_{m+6}=F_{n}-F_{m}=8^{k}-1$ or $2^{k}-1$, which are impossible.

Corollary 3.1.The largest repdigits in base $b$, which can be written as the difference of two Fibonacci numbers are

$$
\begin{array}{ll}
F_{9}-F_{4}=34-3=31=(11111)_{2}, & F_{14}-F_{7}=377-13=364=(111111)_{3}, \\
F_{14}-F_{7}=377-13=364=(222)_{4}, & F_{9}-F_{4}=34-3=31=(111)_{5}, \\
F_{11}-F_{4}=89-3=86=(222)_{6}, & F_{13}-F_{5}=233-5=228=(444)_{7}, \\
F_{10}-F_{2}=55-1=54=(66)_{8}, & F_{14}-F_{7}=377-13=364=(444)_{9},
\end{array}
$$

and

$$
F_{15}-F_{10}=610-55=555=(555)_{10} .
$$

Corollary 3.2. The largest Fibonacci number which can be written as a sum of a repdigit and a Fibonacci number is $F_{15}=610=555+55=555+F_{10}$.

\section{Acknowledgments}

We thank the referee for his/her suggestions which improved the quality of this paper. 


\section{References}

[1] Baker A, Davenport H. The equations $3 x^{2}-2=y^{2}$ and $8 x^{2}-7=z^{2}$. Q. J. Math., Oxford Ser., 1969, 20(1): 129-137.

[2] Bravo J J, Gomez C A, Luca F. Powers of two as sums of two k-Fibonacci numbers. Miskolc Math. Notes, 2016, 17(1): 85-100.

[3] Bugeaud Y, Mignotte M, Siksek S. Classical and modular approaches to exponential Diophantine equations I, Fibonacci and Lucas perfect powers. Ann. Math., 2006, 163(3): 9691018.

[4] De Weger B M M. Algorithms for Diophantine Equations. CWI Tracts 65, Stichting Maths, Centrum, Amsterdam, 1989.

[5] Dujella A, Pethò A. A generalization of a theorem of Baker and Davenport. Q. J. Math., 1998, 49(3): 291-306.

[6] Erduvan F, Keskin R. Repdigits as products of two Fibonacci or Lucas numbers. Proc. Indian Acad. Sci. Math. Sci., 2020, 130(1): 14 pp.

[7] Erduvan F, Keskin R, Şiar Z. Repdigits base b as products of two Fibonacci numbers. Indian J. Pure Appl. Math., 2021, 52(3), 861-868.

[8] Erduvan F, Keskin R, Şiar Z. Repdigits base b as products of two Lucas numbers. Quaest. Math., 2021, 44(10): 1283-1293.

[9] Koshy T. Fibonacci and Lucas Numbers With Applications. Wiley-Interscience Pub, New York, 2001.

[10] Luca F. Fibonacci and Lucas numbers with only one distinct digit. Portugal Math., 2000, 57(2): 243-254.

[11] Luca F. Repdigits as sums of three Fibonacci numbers. Math. Commun., 2012, 17 (1): 1-11.

[12] Matveev E M. An Explicit lower bound for a homogeneous rational linear form in the logarithms of algebraic numbers II. Izv. Ross Akad. Nauk. Ser. Mat., 2000, 64(6): 125-180 (Russian). Translation in Izv Math, 2000, 64(6): 1217-1269. 